\title{
Determining Project Contingency Reserve Using a Fuzzy Arithmetic-Based Risk Analysis Method
}

\author{
Seyed Hamed Fateminia \\ University of Alberta \\ fatemini@ualberta.ca
}

\author{
Nasir Bedewi Siraj \\ University of Alberta \\ siraj@ualberta.ca
}

\author{
Aminah Robinson Fayek \\ University of Alberta \\ aminah.robinson@ualberta.ca
}

\author{
Andrew Johnston \\ University of Alberta \\ amj@,ualberta.ca
}

\begin{abstract}
Traditional techniques for estimating contingency reserve fail to capture subjective uncertainties and expert knowledge, and they rely on historical data. This paper proposes a fuzzy risk analysis model (FRAM) that uses fuzzy arithmetic to analyze risk and opportunity events and determine construction project contingency reserve. The FRAM allows experts to use natural language to assess the probability and impact of risk and opportunity events by employing linguistic scales represented by fuzzy numbers, thus addressing the data reliance problem of probabilistic methods. It enables experts to customize linguistic scales and fuzzy numbers for different project types and stages. The FRAM also deals with the challenges associated with deterministic approaches by addressing measurement imprecision and the subjective uncertainty of experts' opinions. Moreover, the FRAM allows analysts to estimate contingency at different levels of confidence. This paper also illustrates Fuzzy Risk Analyzer ${ }^{\mathcal{O}}\left(F R A^{\mathscr{O}}\right)$, software that implements the fuzzy arithmetic procedure of the FRAM.
\end{abstract}

\section{Introduction}

Risk management is an integral part of project management in the construction industry. A project risk is defined as an uncertain event or condition that has a positive effect (opportunity) or negative effect (threat) on one or more project objectives, such as scope, schedule, cost, or quality [1]. Risk management involves planning risk management activities, identifying potential risks, analyzing risks with qualitative and quantitative techniques, planning and implementing response strategies, and continuous monitoring and control of risks. Quantitative risk analysis is essential for analyzing the combined effects of random occurrences of events and developing a synthesized view of the overall impacts of events on project objectives [2]. Quantitative risk analysis approaches use quantitative methods to analyze the probability of occurrence of risk events and their respective impacts on project objectives and determine the contingency reserve required to mitigate risks or enhance opportunities [3]. One of the primary elements that contributes to the successful achievement of project objectives is the improvement of cost estimation techniques, including contingency calculation [4]. Contingency is a reserve budget added to the estimated project cost baseline to handle risks and uncertainties and help keep the project on budget [5].

Ahmadi-Javid et al. [6] categorize risks into two groups: (1) unknown unknowns that are handled with management reserve and (2) known unknowns that are addressed proactively (i.e., using avoidance, mitigation, and transfer strategies for risks and exploiting, enhancing, and sharing strategies for opportunities) or reactively (i.e., using active and passive acceptance). All risks that are dealt with using proactive strategies or active acceptance strategies are handled with contingency reserve. Risks and opportunities that are addressed with passive acceptance strategies are handled with management reserve [1, 3]. Accurate contingency estimation plays a vital role in achieving both project objectives and efficient management of organizational resources [4]. In the literature, there are several definitions of contingency. The Project Management Institute (PMI) defines contingency reserve as the budget allocated to the schedule or cost baseline for handling identified risks with active response strategies [1]. AACE International defines contingency as "an amount added to an estimate to allow for items, conditions, or events for which the state, occurrence, or effect is uncertain and that experience shows will likely result, in aggregate, in additional costs" [7]. The definition of contingency provided by AACE International excludes costs incurred through major scope changes, force majeure, management reserves, and escalation and currency effects. Schneck et al. [8] categorize contingency as schedule contingency and cost contingency. This paper adopts PMI's definition of contingency and focuses only on cost contingency. Classical techniques for estimating 
contingency reserve are unable to properly analyze risk events because of lack of consideration of uncertainty or lack of historical data to quantitatively assess uncertainty. Deterministic approaches, which require a crisp number to evaluate the probability and impact of risks, rely on expert intuition and experience and do not adequately capture uncertainty $[9,10]$. On the other hand, probabilistic approach, such as Monte Carlo simulation (MCS) and decision tree analysis (DTA), rely heavily on historical data, and the contingency value can therefore be affected by historical data lacking in both quality and quantity [11]. Moreover, both deterministic and probabilistic approaches are incapable of modeling subjective uncertainty.

Fuzzy-based methods, which are based on fuzzy set theory, overcome the limitations of deterministic and probabilistic approaches as they model subjective and imprecise information [12, 13]. Using membership functions (MBF), linguistic variables representing the probability and impact of risks can be mathematically translated into numeric form $[12,14]$. Therefore, fuzzy logic can capture uncertainties resulting from linguistic approximation and imprecise measurement in risk assessment, especially when precise data are not available and there is a reliance on expert knowledge [15].

This paper has three objectives: (1) reviewing traditional techniques for determining contingency reserve and identifying their shortcomings; (2) presenting a fuzzy risk analysis model (FRAM), based on fuzzy arithmetic procedures, for determining contingency; and (3) illustrating a software tool, Fuzzy Risk Analyzer ${ }^{\odot}\left(\right.$ FRA $\left.^{\odot}\right)$, that implements systematic fuzzy arithmetic procedures to determine optimized contingency.

The rest of this paper is organized as follows. First, contingency determination methods are presented in detail, followed by a discussion about the application and benefits of employing fuzzy logic in risk analysis and contingency determination processes. Second, a fuzzy arithmetic-based risk analysis model for determining the contingency reserve of construction projects is proposed. Third, a hypothetical case study is provided to show how the FRAM can be implemented in practice. Finally, conclusions are presented, and future extensions of current research are discussed.

\section{Overview of traditional methods for determining contingency reserve}

There are several methods for determining contingency reserve, including expert judgment, MCS, expected monetary value (EMV), program evaluation and review technique (PERT) [3], predetermined guidelines, and parametric modeling [7]. Baccarini [9] mentions regression analysis and artificial neural networks as prominent alternative methods. Methods for determining contingency reserve can be categorized into two main groups: deterministic and probabilistic.

Deterministic methods are widely employed on construction projects due to their simplicity $[1,9]$ and transparency [16]. In deterministic methods, either a predefined percentage is added to the project cost baseline or the probability and impact of risks are assessed using a single-point estimate or linguistic expressions in a probability-impact matrix (PI Matrix) to determine their severity [5]. Fixed percentages are determined for different types and phases of projects and can be a single value or a range of values. Deterministic approaches are unable to address uncertainties effectively [17]; consider the unique effects of project complexity, market condition, and location [18]; and assess risks properly [10, 19]. Moreover, they do not provide a confidence level for the sufficiency of the estimated contingency.

Uncertainties in probabilistic methods, such as MCS, are explicitly modeled by employing appropriate probability distributions [20]. Probabilistic methods significantly rely on historical data and probability theory [21] and can be categorized as simulation-based methods (e.g., range estimating, integrated cost and schedule) and non-simulation-based methods (e.g., probability tree, PERT, analytic hierarchy process [AHP], expected value, regression) [5]. Probabilistic methods are unable to determine accurate contingency when historical data is unavailable or inappropriate. Moreover, probabilistic methods assume that variations in costs are strictly random in nature.

Fuzzy-based methods [12], are suitable for handling the subjectivity and imprecision inherent in human assessments and it addresses the aforementioned limitations of deterministic and probabilistic methods. With fuzzy logic, instead of using single values for risk probabilities and impacts, experts can provide their assessments using linguistic terms such as "very low," "medium," "high," etc., which are in turn represented by fuzzy numbers [22]. Fuzzy numbers are a special type of fuzzy sets used for representing the values of realworld parameters when exact values cannot be measured due to inappropriate information or a lack of knowledge. Subjective assessments of the problem can thereby be used to derive an acceptable approximation [23]. The literature review shows that there is an increasing tendency to address the limitations of traditional risk analysis techniques by integrating them with fuzzy logic.

Iranmanesh et al. [24] proposed a fuzzy expert system (FES), including two-layered fuzzy inference, to assess risk events of software projects. The first layer of 
their model evaluates the qualitative risk exposure of 12 risk factors based on a $5 \times 5$ risk matrix consisting of 25 fuzzy IF-THEN rules (e.g., risk of factor 1 is SIGNIFICANT). In the second layer, which is a multiinput single-output inference system, the total risk of the project is determined by evaluating the outputs of the first layer based on 17 million $\left(4^{12}\right)$ rules, which are reduced to almost 4000 rules for each input by employing heuristic programming. A large number of rules makes the risk assessment process infeasible, especially in the case of a large number of risk factors, which limits the applicability of this model for risk analysis in construction. Skorupski [25] developed a risk assessment method by integrating simulation analysis of the probability of a risk of an air traffic accident with a fuzzy analysis of its effects. A fuzzy risk matrix was employed in which the probability and severity of the effects are represented by linguistic variables, and a fuzzy inference system performs the risk assessment. Sadeghi et al. [26] suggested a method of dealing with both random and subjective uncertainties in determining project contingency by integrating fuzzy set theory with Monte Carlo simulation. However, the proposed method is unable to estimate the individual effect of each risk event; rather, it determines the range estimate of the combined effect of risk events. Nasirzadeh et al. [27] integrated fuzzy logic with system dynamics to capture interdependencies and interactions among different risks and variables using feedback loops. However, having several variables in the model makes it difficult to develop the feedback loops and establish the mathematical equations. Another approach used to develop a fuzzy hybrid model for risk analysis includes integrating failure mode and effect analysis (FMEA), AHP, and fuzzy logic to assess risks and determine contingency [23]; however, this approach requires significant effort to establish clearly defined linguistic definitions for cost, time, and quality impacts along with probability of occurrence, detection, and level of criticality of risk events. Moreover, it is unable to consider opportunities and deal with multiple failure scenarios. Another method prioritized the risk factors of a project using the AHP and fuzzy set theory [28]; however, this approach involves lengthy and laborious pairwise comparisons and is unable to incorporate new information into the risk structure. Abdelgewad et al. [29] integrated fault tree analysis (FTA) and fuzzy set theory for the quantitative assessment of risk events; however, this method is not capable of capturing all scenarios and modeling correlation between risks.

Although various research projects have been carried out to address the limitations of existing risk analysis methods, there is a knowledge gap when dealing with subjective uncertainties and imprecisions in determining contingency. This paper proposes a FRAM based on fuzzy arithmetic procedures, for analyzing risk events and determining the contingency reserve of construction projects effectively and efficiently. In addition, this paper discusses a software tool called FRA ${ }^{\odot}$ that has been developed to implement the model.

\section{Fuzzy arithmetic-based risk analysis model (FRAM)}

The steps and their outputs of the FRAM are illustrated in Figure 1 and are detailed in the following subsections.

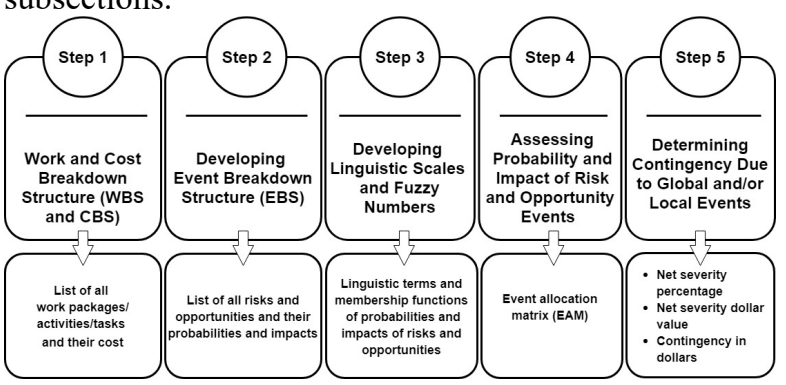

Figure 1. Steps and their outputs of FRAM

\subsection{Developing work and cost breakdown structures}

The work breakdown structure (WBS), a hierarchical decomposition of the total scope of work, is considered the foundation of the FRAM. It is assumed that each project has up to a three-level WBS comprising work package, activity, and task carried out by the project team to accomplish project objectives. PMI [1] defines a work package as "the work defined at the lowest level of the work breakdown structure for which cost and duration are estimated and managed" and activity as "a distinct, scheduled portion of work performed during a project". Figure 2 shows a wind farm project comprising a two-level WBS in FRA ${ }^{\circ}$. Once the WBS is established, the cost breakdown structure (CBS) must be developed to determine the cost of work packages/activities/tasks.

\subsection{Developing the event breakdown structure}

Identifying which potential risk and opportunity events affect project objectives is the second step in the FRAM. Risk and opportunity events in construction can be identified using a combination of varying 


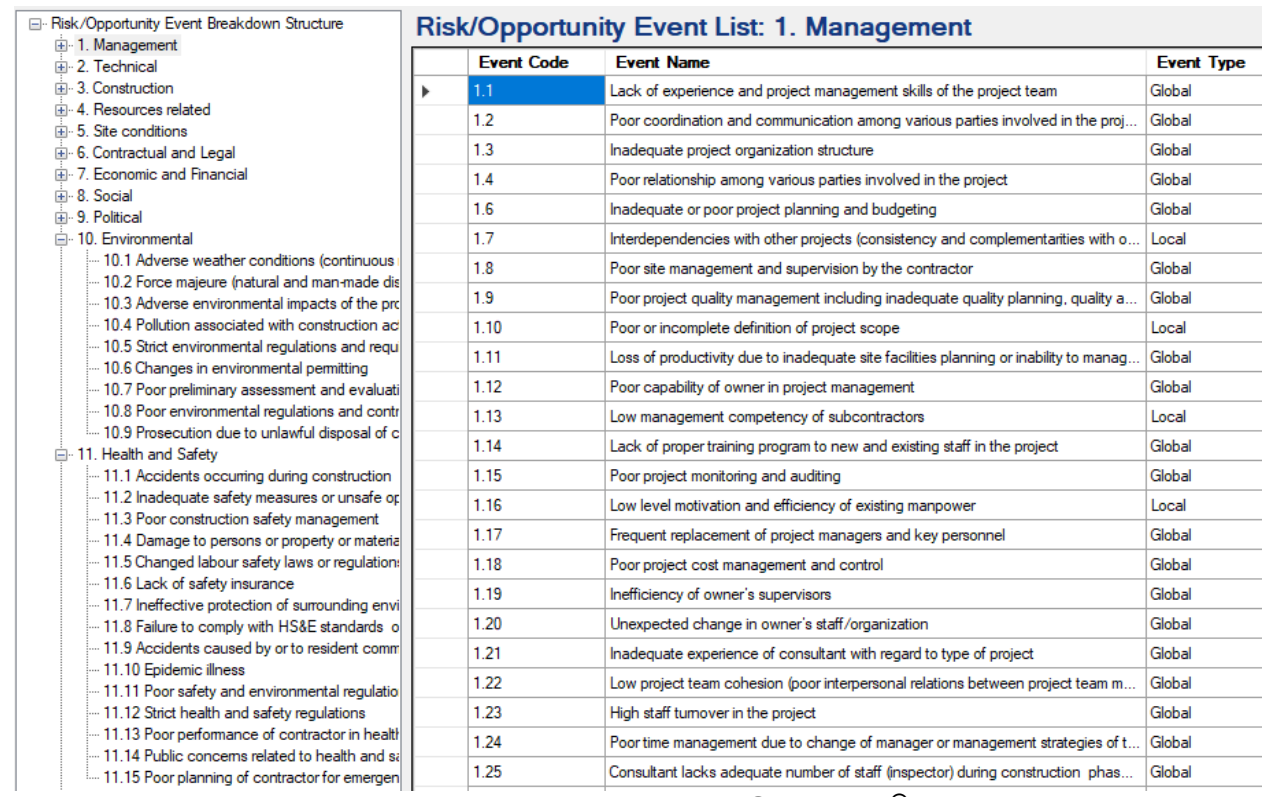

Figure 2. Two-level EBS in FRA ${ }^{\odot}$

information gathering techniques and diagramming and analysis-based techniques. The literature indicates that there is no standard or consensus on the categorization of risk and opportunity events in the construction industry, and as a result, a variety of approaches have been recommended [30]. The default event breakdown structure (EBS) template in the FRAM was developed based on a systematic review and detailed content analysis conducted by Siraj and Fayek [31].

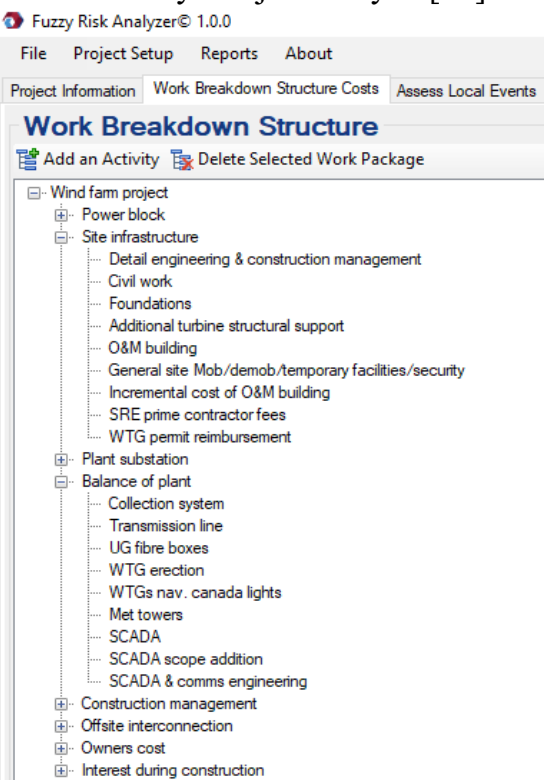

Figure 3. Two-level WBS comprising work package and activity in $\mathrm{FRA}^{\odot}$

The systematic and content analysis was done on 130 selected articles from well-regarded journals in construction engineering and management published between 1990 and 2017. Siraj and Fayek [31] identified numerous common risk and opportunity events in construction and categorized them into eleven groups: management, technical, construction, resource-related, site conditions, contractual and legal, economic and financial, social, political, environmental, and health and safety. This classification, which is illustrated in Figure 3, is adopted in the FRAM as the basis to develop an initial two-level EBS. In the FRAM, the main categories and subcategories of the EBS as well as the event types can be customized for different project types.

\subsection{Developing linguistic scales and fuzzy numbers}

In the third step, linguistic terms and scales are created to assess the probability and impact of risks and opportunities. Each linguistic term is represented by triangular or trapezoidal fuzzy numbers, in which single crisp values used in deterministic methods are replaced for assessing the probability and impact of risk and opportunity events.

Triangular and trapezoidal shapes are the most commonly used shapes for fuzzy numbers whose supports are an open interval of real numbers [32, 33]. The concept of trapezoidal fuzzy numbers is illustrated in Figure 4, using four parameters $a, b, c$, and $d$, where $b$ and $c$ indicate the lower and upper modal values, respectively, of the core (i.e., the set of all elements of the universe of discourse that have a membership degree of 1 in the fuzzy number) and $a$ and $d$ are the lower and 
upper bounds, respectively, of the support (i.e., the set of all elements of the universe of discourse that have a non-zero membership degree in the fuzzy number). A special case of trapezoidal fuzzy numbers occurs when $b=c$, resulting in a triangular fuzzy number. Wide support of a fuzzy number represents a higher level of uncertainty [34]. Fuzzy numbers are context-dependent, and parameters can differ based on the application and the data describing the problem under consideration.

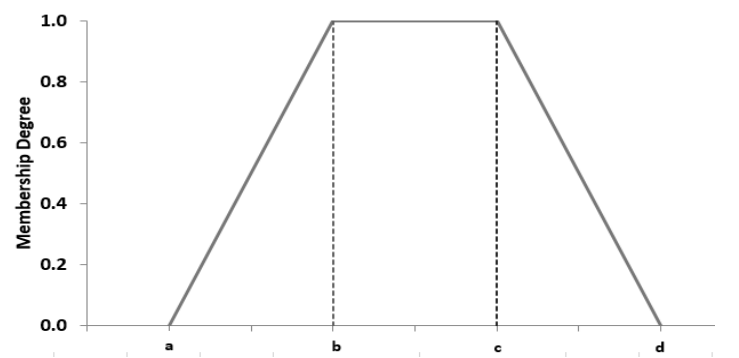

Figure 4. Example of trapezoidal fuzzy number

In the FRAM, the probability and impact of risk and opportunity events are defined using five linguistic terms (i.e., very low, low, medium, high, and very high) as recommended by Hall [35]. Figure 5 shows the default linguistic terms and their respective triangular membership functions for risk probability and opportunity probability. Figure 6 depicts the trapezoidal MBFs representing the linguistic terms for risk impact and opportunity impact.

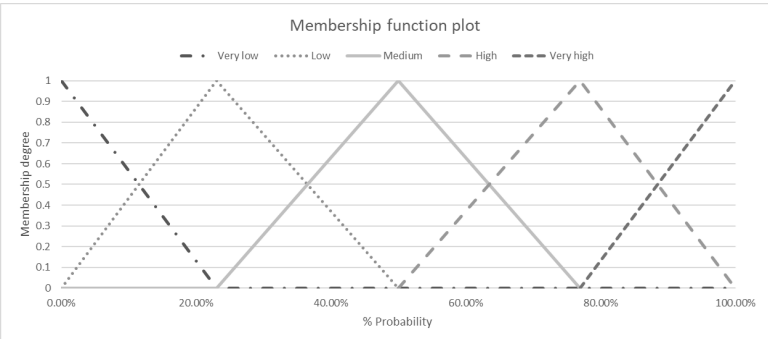

Figure 5. MBFs for risk probability and opportunity probability

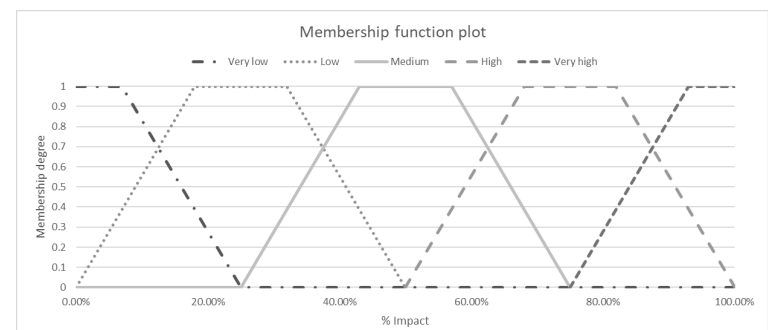

Figure 6. MBFs for risk impact and opportunity impact

\subsection{Assessing probability and impact and developing the event allocation matrix}

Following the identification of events and the creation of linguistic terms and scales, an event allocation matrix (EAM) can be employed to specify the relationships among the events and the project's work packages/activities/tasks based on expert judgment and project context. Each event can be evaluated simultaneously as a risk and an opportunity. In the FRAM, risk and opportunity events are identified by the risk analyst as either global or local. Global events are risk and opportunity events that impact several work packages/activities/tasks simultaneously and are assessed for the group of assigned work packages/activities/tasks. Local events are risk and opportunity events that are assigned individually to individual work packages/activities/tasks and can be assessed individually for each work package/activity/task. The EAM technique also enables experts to determine the percentage value (ranging from 0 to 100 percent) of each work package/activity/task impacted by a given local or global risk and opportunity event. The portion of the estimated cost of the work package/activity/task that is affected by a local or global event is determined in FRA $^{\odot}$, the software tool that implements the steps of FRAM, in the form of either a percentage or a dollar value of the estimated cost of the corresponding work package/activity/task.

\subsection{Determining work package/activity/task and project contingency}

In the final step, work package/activity/task contingencies due to local risk and opportunity events is calculated using the fuzzy arithmetic procedure as follows.

1. The level (work package, activity, or task) at which the risk assessment should be carried out is decided by the experts. Then, the local events affecting each work package/activity/ task are determined using the EAM and the probability and impact of the events are assessed by experts based on the established linguistic scales. Local events are assessed two times to ensure that both risk and opportunity events are considered: one assessment assumes they may result in a risk event (using probability and impact scales for risk) and the other assumes it may lead to an opportunity event (using probability and impact scales for opportunity).

2. For any local event affecting a specific work package/activity/task, risk and opportunity 
severity as a percentage is determined by multiplying the fuzzy numbers representing its probability and impact.

3. The net severity percentage attributed to each local risk event is equal to the percentage of opportunity severity minus the percentage of risk severity as determined in step 2 .

4. The net severity dollar value (a fuzzy number) attributed to each local risk event is determined by multiplying its net severity percentage by the cost of the work package/activity/task affected by the corresponding event.

5. The contingency in dollars of the work package/activity/task is calculated as a fuzzy number by adding the net severity in dollars of all local events affecting that work package/activity/task.

To determine project contingency due to global risk and opportunity events, the procedure described above must be followed. A percentage of the estimated cost of each work package/activity/task affected by a global event is determined by experts. Subsequently, the abovementioned procedure for local risks and opportunities is implemented to assess the probability and impact of each global risk event for the affected group of work packages, activities, and tasks, rather than for each work package/activity/task individually. Final fuzzy numbers that represent both local and global contingencies of individual work packages, in dollars, are added together to determine overall project contingency.

\section{Fuzzy arithmetic operations in the FRAM}

A fuzzy set class was developed in the C\# programming language to perform fuzzy arithmetic operations in the FRAM. The fuzzy set class has the capability to define a fuzzy set that is a triangular or trapezoidal shape, taking its $\alpha$-cuts, performing fuzzy arithmetic operations, and graphically representing the fuzzy number [36]. Moreover, the fuzzy set class has the ability to determine a representative value of the fuzzy number using different defuzzification methods and confidence levels [37,38]. A horizontal discretization technique proposed by Hanss [39] is adopted in the fuzzy set class to define a fuzzy set based on its $\alpha$-cuts. Two methods are applied to implement fuzzy arithmetic operations in the fuzzy set class: (1) the traditional horizontal $\alpha$-cut method (standard fuzzy arithmetic) and (2) the extension principle based on $t$-norms (extended fuzzy arithmetic).

In standard fuzzy arithmetic, interval analysis is generalized and input fuzzy numbers are discretized into several $\alpha$-cuts, and interval calculations are implemented on each $\alpha$-level cut of the inputs to obtain the $\alpha$-cut of the output. Then the union of the $\alpha$-cuts is employed to create the final fuzzy set according to the representation theorem. Eq. 1 shows the mathematical representation of standard fuzzy arithmetic.

$\mathrm{C}(\mathrm{z})=\mathrm{A}(x) \circledast \mathrm{B}(\mathrm{y})=\sup _{\alpha \in[0,1]} \alpha\left(\left(A_{\alpha} * B_{\alpha}\right)(z)\right)$,

where $C(z)$ is the output fuzzy number, $A(x)$ and $B(y)$ are input fuzzy numbers, and $\mathrm{A}_{\alpha}$ and $\mathrm{B}_{\alpha}$ are considered the $\alpha$-cuts of the input fuzzy numbers. In this fuzzy class, ten $\alpha$-cuts, $\alpha=\{0,0.1,0.2,0.3, \ldots, 1\}$, are used to discretize the fuzzy sets. In the equation, $\circledast$ represents any type of the four basic arithmetic operations. For example, for a risk with probability and impacts of $A_{l}$ and $A_{2}$, respectively, where $A_{1}$ and $A_{2}$ are fuzzy numbers, the percentage severity $\mathrm{B}$ is calculated as shown in Eq. 2.

$\mathrm{B}_{\alpha}=\mathrm{A}_{1 \alpha} \times \mathrm{A}_{2 \alpha}$

The standard fuzzy arithmetic method is widely used in the computing of fuzzy arithmetic operations. However, it can lead to an accumulation of fuzziness, which causes the overestimation of uncertainty [39]. Accordingly, the use of extended fuzzy arithmetic is preferred in some cases. Extended fuzzy arithmetic, proposed by Zadeh [12], is a generalized version of standard arithmetic on real numbers to fuzzy numbers which requires a pointwise calculation among the fuzzy sets. Extended fuzzy arithmetic calculates the membership degree of each output by taking the supremum of the $t$-norms of the membership degrees of the input points used to determine that output. A general form of extended fuzzy arithmetic is defined in Eq. 3.

$\mathrm{C}(\mathrm{z})=\mathrm{A}(x) \circledast \mathrm{B}(\mathrm{y})=\sup _{z=x * y}(t(A(x), B(y)))$,

where $t$ is any $t$-norm operator on fuzzy sets, $C(z)$ is the output fuzzy number, and $A(x)$ and $B(y)$ are input fuzzy numbers. Extended fuzzy arithmetic can be applied by four common fuzzy $t$-norm operators-minimum, algebraic product, bounded difference, and drastic product - which are different in terms of strength and continuity. Although in terms of strength the highest $t$ norm is the minimum and the lowest is the drastic product, there is no agreed-upon rule to order fuzzy $t$ norms in terms of their strength [15]. The results of extended fuzzy arithmetic using the minimum $t$-norm are similar to those obtained using standard fuzzy arithmetic. Therefore, the implementation of extended fuzzy arithmetic using any $t$-norm other than the minimum can decrease the overestimation of uncertainty. The continuity of a $t$-norm represents the 
sensitivity of output changes to input changes and can be specified by employing the functions that define the $t$-norm. Therefore, continuous $t$-norms lead to more accurate results for fuzzy operations in making decisions, modeling systems, and optimizing problems. Minimum, algebraic product, and bounded difference $t$ norms are continuous, whereas the drastic product $t$ norm is non-continuous [32]. Lin et al. [40] recommend the use of the drastic product $t$-norm in fuzzy arithmetic as it is easy to use in fuzzy arithmetic operations and it has the ability to control the growth of uncertainty during calculations. Minimum, algebraic product, drastic product, and bounded difference $t$-norms are all implemented in the fuzzy set class.

Finally, the fuzzy contingency value can be represented as a crisp value using the single value (defuzzification) method or the interval value using the confidence level. Figure 7 shows the single value (defuzzification) methods available in $\mathrm{FRA}^{\odot}$, including center of area (COA), smallest of maxima (SOM), middle of maxima (MOM), and largest of maxima (LOM). The COA is the $\mathrm{x}$-axis value that corresponds to the center of area of the fuzzy number; it is the best representation of the shape of the output fuzzy number. The SOM, MOM, and LOM are the smallest, middle, and largest of the range of $\mathrm{x}$-axis values with the largest membership degree, respectively.

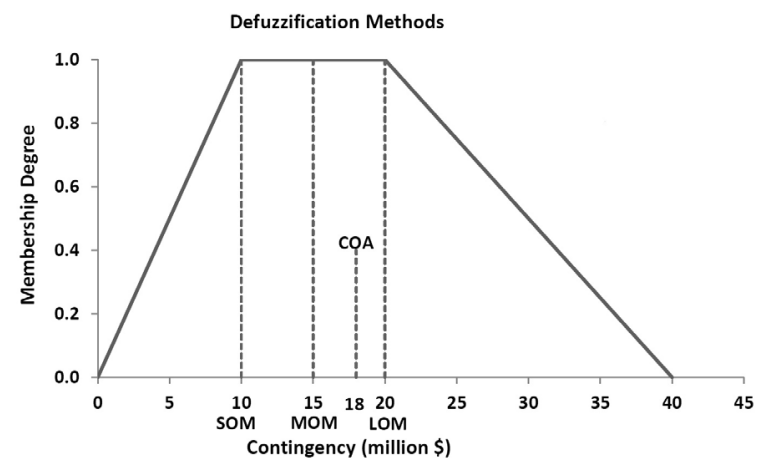

Figure 7. Defuzzification methods available in FRA $^{\odot}$

There is no standard or guideline for selecting the most appropriate single value (defuzzification) method for all types of projects. The defuzzification method can be selected based on project context, the preferences of the risk analyst, and the risk attitude of the analyst. For instance, the SOM and LOM should be employed to get optimistic and pessimistic contingency values, respectively. To determine the performance of the defuzzification methods, the results from the model for a given project should be compared to the actual cost of the project.

The confidence level represents the level of confidence associated with the range of contingency values determined from the corresponding $\alpha$-cut level (possibility degree), and it ranges from 0 to 1 . The possibility degree is equal to 1 minus the confidence level $(1-$ confidence level). For the example shown in Figure 8 , there is a possibility degree of 0.6 that the contingency values for the project fall within the range of 6 and 28 million dollars. The corresponding confidence level associated with this range of values is 0.4 .

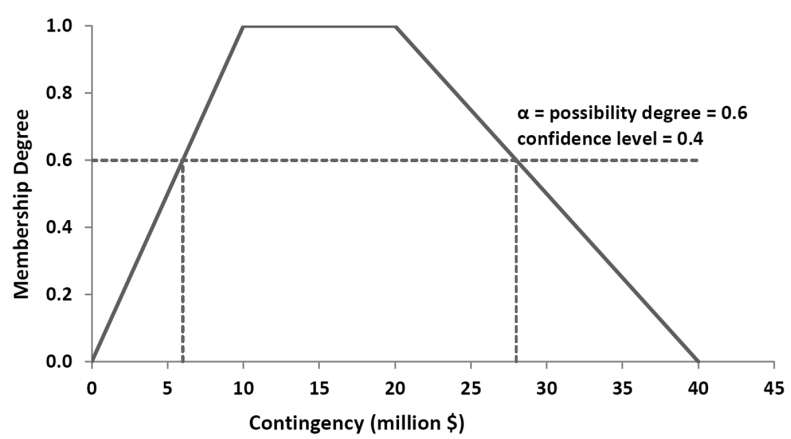

Figure 8. Contingency range based on confidence level and possibility degree

\section{Implementation of the FRAM in FRA $\odot$}

This section presents a simplified extraction of a real-world case as an illustration of how the FRAM can be implemented in practice. $\mathrm{FRA}^{\odot}$ is used to implement the systematic fuzzy arithmetic procedures proposed in the FRAM.

A hypothetical wind farm project valued at approximately $\$ 556$ million is used as the example. Since the work breakdown structure is the basis of risk analysis in the FRAM, a list of work packages/activities/tasks must first be developed from the project master plan. This list is used as an input to the cost estimation process that establishes the cost breakdown structure, including the cost of each work package/activity/task. The example project had 8 work packages and 73 activities, and the analysis was done at the work package level. Table 1 presents the work packages and their respective costs.

Table 1. Cost of work packages

\begin{tabular}{ll}
\hline Work package name & Total cost $(\$)$ \\
\hline Power block & $326,650,000$ \\
Site infrastructure & $61,800,000$ \\
Plant substation & $17,750,000$ \\
Balance of plant & $52,640,000$ \\
Construction management & $5,565,000$ \\
Off-site interconnection & $33,750,000$ \\
Owner's cost & $42,420,000$ \\
Interest during construction & $15,200,000$ \\
\hline
\end{tabular}




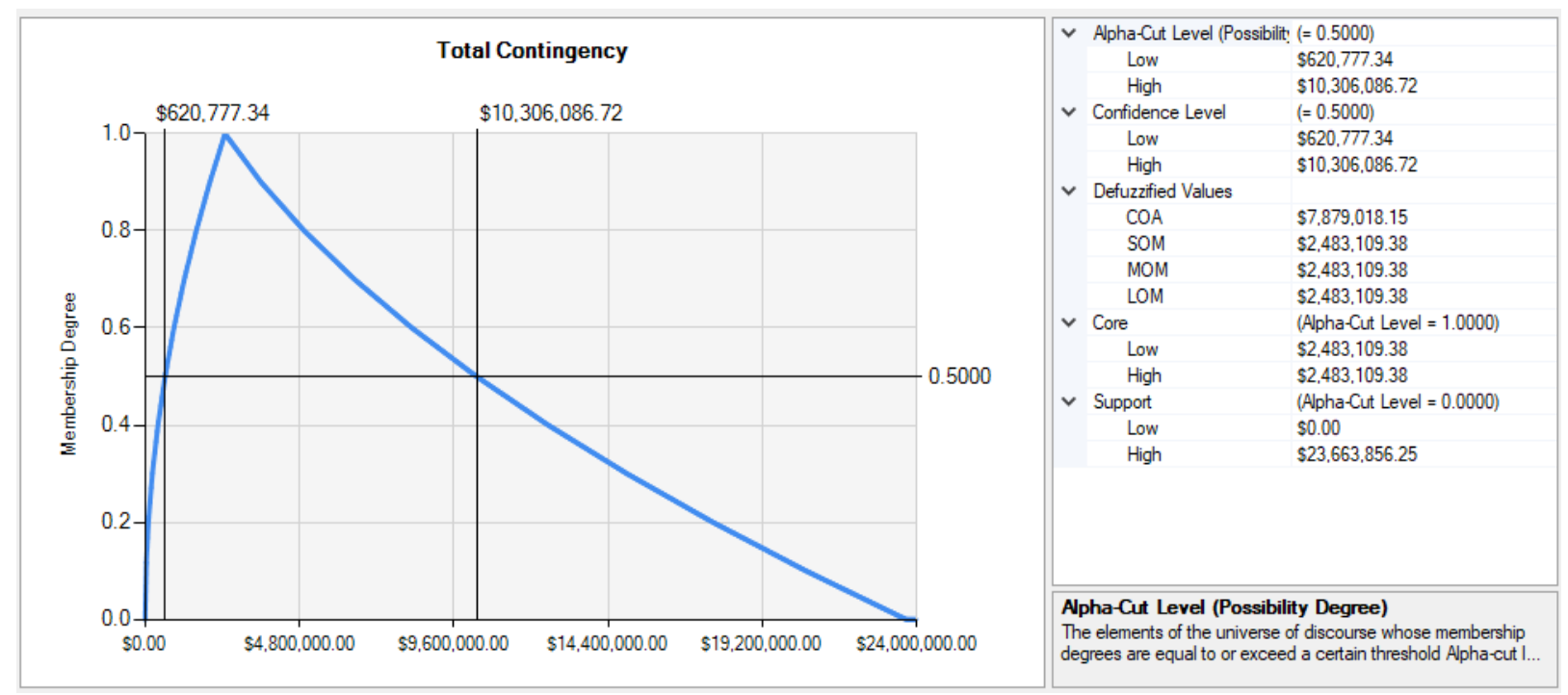

Figure 9. Total contingency of the project

The second step is to modify the EBS for the specific project under consideration.

The default EBS, shown in Figure 2, was modified. The final EBS has 18 risks and opportunities, including 4 global and 14 local risk events. In the third step, the probability and impact linguistic scales were developed for the identified risk and opportunity events, and their respective fuzzy numbers were generated employing the modified horizontal approach coupled with curve fitting. Historical cost variance data related to work packages/activities/tasks from similar previously completed projects were examined to verify the linguistic scales for the impact of the project's risks and opportunities. In the fourth step, local and global risks and opportunities were assigned to relevant work packages to create the EAM, assess the probability and impact of local and global risks and opportunities, and determine the percentage of work package costs impacted by the events. Finally, fuzzy arithmetic was applied to determine work package and project contingency using $\mathrm{FRA}^{\odot}$. $\mathrm{FRA}^{\odot}$ enables the user to select either standard fuzzy arithmetic or extended fuzzy arithmetic based on the four $t$-norms. Figure 9 depicts the fuzzy number that represents the total contingency of the wind farm project. The defuzzified value of project contingency based on the COA method is $\$ 7,879,018.15$, whereas the contingency value based on the SOM, MOM, and LOM is $\$ 2,483,109.38$. Figure 9 also shows that at an $\alpha$-cut level of 0.50 , there is a confidence level of 0.5 (possibility of 0.5 ) that the project contingency will be between $\$ 620,777.34$ and $\$ 10,306,086.72$.

\section{Discussion}

The FRAM is flexible, as it enables experts to customize the linguistic scales and fuzzy numbers for different project types and stages. It also provides risk analysts with further flexibility in terms of its contingency calculation and output determination methods by enabling users to set contingency based on their risk attitude. Moreover, the FRAM allows risk analysts to estimate contingency in terms of confidence intervals at different levels of confidence.

Table 2 presents a comparison of FRAM with other risk assessment and contingency methods. The FRAM deals with the challenges associated with deterministic approaches by addressing measurement imprecision and the subjective uncertainty of experts' opinions in assessing the probability and impact of risks. Unlike the PI matrix and predefined percentages, FRAM is a quantitative method that is able to address subjective uncertainties in assessing risks and opportunities, considers both global and local risks and opportunities in work packages/activities/tasks, and provides a confidence level for the estimated contingency. The FRAM uses a fuzzy arithmetic procedure that addresses the limitations of probabilistic methods such as MCS, including substantial reliance on historical data and a lack of consideration of subjective uncertainty, by employing expert judgment, linguistic scales, and fuzzy numbers to assess risks and opportunities.

FRAM also addresses the disadvantages of hybrid methods. In comparison with fuzzy AHP, the FRAM does not require lengthy and laborious pairwise 
Table 2. Comparison of FRAM with other risk assessment and contingency methods

\begin{tabular}{|c|c|c|c|c|c|c|c|c|c|c|}
\hline & Criteria & 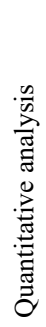 & 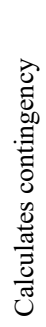 & $\begin{array}{l}\frac{y}{0} \\
0 \\
0 \\
0 \\
0 \\
0 \\
0 \\
0 \\
0 \\
0\end{array}$ & 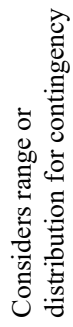 & 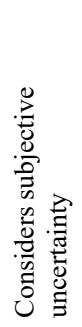 & $\begin{array}{l}\bar{d} \\
\frac{0}{0} \\
0 \\
0 \\
\frac{0}{0} \\
0 \\
0 \\
0 \\
0 \\
0 \\
0 \\
0 \\
0\end{array}$ & 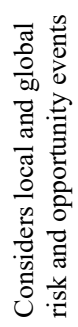 & 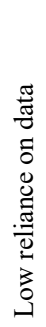 & 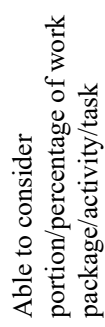 \\
\hline \multirow{2}{*}{$\begin{array}{l}\text { Deterministic } \\
\text { approach }\end{array}$} & Probability-impact matrix (PI matrix) & - & $\sqrt{ }$ & $\sqrt{ }$ & - & - & - & - & - & - \\
\hline & Predefined percentages & - & $\sqrt{ }$ & - & - & - & - & - & - & - \\
\hline $\begin{array}{c}\text { Probabilistic } \\
\text { approach }\end{array}$ & Monte Carlo simulation (MCS) & $\sqrt{ }$ & $\sqrt{ }$ & $\sqrt{ }$ & $\sqrt{ }$ & - & $\sqrt{ }$ & - & - & - \\
\hline \multirow{4}{*}{$\begin{array}{l}\text { Fuzzy-based } \\
\text { approach }\end{array}$} & $\begin{array}{l}\text { Fuzzy analytic hierarchy process } \\
\text { (Fuzzy AHP) }\end{array}$ & - & - & $\sqrt{ }$ & - & $\sqrt{ }$ & - & - & $\sqrt{ }$ & - \\
\hline & $\begin{array}{l}\text { Fuzzy failure mode and effect } \\
\text { analysis (Fuzzy FMEA) }\end{array}$ & $\sqrt{ }$ & - & $\sqrt{ }$ & - & $\sqrt{ }$ & - & - & $\sqrt{ }$ & - \\
\hline & $\begin{array}{l}\text { Fuzzy fault tree analysis (Fuzzy } \\
\text { FTA) }\end{array}$ & $\sqrt{ }$ & - & $\sqrt{ }$ & - & $\sqrt{ }$ & - & - & $\sqrt{ }$ & - \\
\hline & Fuzzy risk analysis model (FRAM) & $\sqrt{ }$ & $\sqrt{ }$ & $\sqrt{ }$ & $\sqrt{ }$ & $\sqrt{ }$ & $\sqrt{ }$ & $\sqrt{ }$ & $\sqrt{ }$ & $\sqrt{ }$ \\
\hline
\end{tabular}

comparisons, and it has the flexibility to incorporate new information into the risk structure at each step. Unlike fuzzy FMEA, the FRAM does not depend on complex failure mode and effect scenarios that must be solicited from experts. Moreover, fuzzy FMEA and FTA can only prioritize risk events for risk response actions and are unable to quantify the contingency.

\section{Conclusions and future research}

This paper presents a systematic, transparent, and flexible FRAM, based on fuzzy arithmetic procedures, for assessing risk and opportunity events and determining the contingency reserve of construction projects. By employing fuzzy logic, fuzzy arithmetic based on the $\alpha$-cut method, and the extension principle using different t-norms, the FRAM addresses the limitations of traditional risk analysis methods, such as a high reliance on historical data and the inability to account for the subjective uncertainty associated with assessing risk and opportunity events. Different levels of confidence can also be determined for contingency.

A software tool, $\mathrm{FRA}^{\odot}$, has been developed to automate the steps of the FRAM. FRA ${ }^{\odot}$ provides experts with the flexibility to determine work package and project contingencies at different levels of confidence.

Future research will investigate the development of a framework to aggregate expert assessments by accounting for the varying expertise levels of experts' in risk management. Future research will also focus on validation of the FRAM using real project data and comparing results with traditional contingency determination methods. In addition, linguistic scales for different project types and contexts will be developed to improve the accuracy of risk analysis.

\section{Acknowledgements}

This research is funded by the Natural Sciences and Engineering Research Council of Canada (NSERC) Industrial Research Chair in Strategic Construction Modeling and Delivery (NSERC IRCPJ 428226-15), which is held by Dr. Aminah Robinson Fayek. The authors gratefully acknowledge the financial support provided by industry partners and NSERC through the Chair.

\section{References}

[1] A Guide to the Project Management Body of Knowledge (PMBOK ${ }^{\circledR}$ guide) - Sixth edition. Project Management Institute, Newtown Square, PA, 2017.

[2] S.H. Fateminia, N. Gerami Seresht, and A.R. Fayek, "Evaluating Risk Response Strategies on Construction Projects Using a Fuzzy Rule-Based System”, 36th International Symposium on Automation and Robotics in Construction, Banff, 2019.

[3] The Standard for Risk Management in Portfolios, Programs, and Projects, Project Management Institute, April 2019.

[4] M. Uzzafer, "A Contingency Estimation Model for Software Projects", International Journal of Project Management, vol. 31, no. 7, pp. 981-993, 2013. 
[5] P. Bakhshi and A. Touran, "An Overview of Budget Contingency Calculation Methods in Construction Industry", Creative Construction Conference, 2014.

[6] A. Ahmadi-Javid, S.H. Fateminia, and H.G. Gemunden, "A Method for Risk Response Planning in Project Portfolio Management", Project Management Journal, (accepted January 2019.

[7] AACE International, "Cost Engineering Terminology", AACE Recommended Practice No. 10S-90, TCM Framework: General Reference, March 2010.

[8] D. Schneck, R. Laver, and M. O'Connor, "Cost Contingencies, Development Basis, and Project Application”, Journal of the Transportation Research Board, no. 2111, pp. 109-124, December 2009.

[9] D. Baccarini, "Estimating Project Cost ContingencyBeyond the 10\% Syndrome", Australian Institute of Project Management National Conference, Australian Institute of Project Management, 2005.

[10] C. Kirchsteiger, "On the Use of Probabilistic and Deterministic Methods in Risk Analysis", Journal of Loss Prevention in the Process Industry, vol. 12, no. 5, pp. 399-419, 1999.

[11] A. Salah and O. Moselhi, "Contingency Modelling for Construction Projects Using Fuzzy Set Theory", Engineering Construction and Architecture Management, vol. 22, no. 2, pp. 214-241, 2015.

[12] L.A. Zadeh, "Fuzzy Sets", Information and Control, vol. 8, no. 3, pp. 338-353, 1965.

[13] N. Gerami Seresht and A.R. Fayek, "Computational Method for Fuzzy Arithmetic Operations on Triangular Fuzzy Numbers by Extension Principle", International Journal of Approximate Reasoning, vol. 106, pp. 172-193, 2019.

[14] J.C. Bezdek, Pattern Recognition with Fuzzy Objective Function Algorithms, Plenum, New York, 1981.

[15] W. Pedrycz and F. Gomide, An Introduction to Fuzzy Sets: Analysis and Design, MIT Press, Cambridge, MA, 2007.

[16] K. Shang and Z. Hossen, "Applying Fuzzy Logic to Risk Assessment and Decision-Making", Casualty Actuarial Society / Canadian Institute of Actuaries / Society of Actuaries Joint Risk Management Section Report, Canada: Casualty Actuarial Society, Canadian Institute of Actuaries, Society of Actuaries, 2013.

[17] S. Mak and D. Picken, "Using Risk Analysis to Determine Construction Project Contingencies", Journal of Construction Engineering and Management, vol. 126, no 2, pp. 130-136, 2000.

[18] A. Olumide, S. Anderson, and K. Molenaar, "Sliding Scale Contingency for the Project Development Process", Journal of the Transportation Research Board, no. 2151, pp. 21-27, August 2010.

[19] M. Toakley, "Risk Management Applications-A Review,' Australian Institute of Building, vol. 6, pp. 77-85, 1995.

[20] A. Touran, "Owners Risk Reduction Techniques Using a CM", CMAA Research Report, Construction Management Association of America, October 2006.

[21] C.P. Robert and G. Casella, Monte Carlo Statistical Methods, 2nd edition, Springer, New York, NY, 2004.

[22] L.A. Zadeh, "The Concept of a Linguistic Variable and Its Application to Approximate Reasoning", Information Science, vol. 8, no. 3, pp. 199-249, 1975.
[23] M. Abdelgawad and A.R., Fayek, "Risk Management in the Construction Industry Using Combined Fuzzy FMEA and Fuzzy AHP", Journal of Construction Engineering and Management, 10.1061/(ASCE)CO.1943-7862.0000210, pp. 1028-1036, 2010.

[24] S. H. Iranmanesh, S. B. Khodadadi and S. Taheri, "Risk Assessment of Software Projects Using Fuzzy Interface System", International Conference on Computers and Industrial Engineering, pp. 1149-1154, 2009.

[25] J. Skorupski. "The Simulation-Fuzzy Method of Assessing the Risk of Air Traffic Accidents Using the Fuzzy Risk Matrix," Safety Science, vol.88, pp. 76-87, 2016.

[26] N. Sadeghi, A.R. Fayek, and W. Pedrycz, "Fuzzy Monte Carlo Simulation and Risk Assessment in Construction", Computer-Aided Civil and Infrastructure. Engineering, vol. 25, pp. 238-252, 2010.

[27] F. Nasirzadeh, A. Afshar, M. Khanzadi, and S. Howick, "Integrating System Dynamics and Fuzzy Logic Modelling for Construction Risk Management", Construction Management Economy, pp. 1-16, 2008.

[28] A. Nieto-Morote and F. Ruz-Vila, "A Fuzzy Approach to Construction Project Risk Assessment", International Journal of Project Management, vol. 29, pp. 220-231, 2011.

[29] M. Abdelgewad and A.R. Fayek, "Fuzzy Reliability Analyzer: Quantitative Assessment of Risk Events in the Construction Industry Using Fault-Tree Analysis", Journal of Construction Engineering and Management, vol. 137, pp. 294302, 2011.

[30] D. Proske, Catalogue of Risks: Natural, Technical, Social and Health Risks, Springer, Berlin, 2008.

[31] N.B. Siraj and A.R. Fayek, "Risk Identification and Common Risks in Construction: Literature Review and Content Analysis", Journal of Construction Engineering and Management, 33 manuscript pages, accepted Feb. 6, 2018.

[32] A.R. Fayek (Ed.), Fuzzy Hybrid Computing in Construction Engineering and Management Emerald Publishing Limited, Bingley, United Kingdom, 2018.

[33] P. Lorterapong and O. Moselhi, "Project-Network Analysis Using Fuzzy Sets Theory", Journal of Construction Engineering and Management., 10.1061/ (ASCE) 07339364(1996)122:4(308), pp. 308-318, 1996.

[34] A. J. Abebe, V. Guinot, and D.P. Solomatine, "Fuzzy Alpha-Cut versus Monte Carlo Techniques in Assessing Uncertainty in Model Parameters", Proceedings of the 4th International Conference on Hydro Informatics, Cedar Rapids, IA, pp. 1-8, 2000.

[35] E.M. Hall, Managing Risk: Methods for Software Systems Development, Addison-Wesley, Reading, MA, 2000. [36] W. Pedrycz. and F. Gomide, An Introduction to Fuzzy Sets: Analysis and Design, MIT Press, Cambridge, MA, 1998. [37] W. Pedrycz and F. Gomide, Fuzzy System Engineering: Toward Human-Centric Computing, John Wiley and Sons, Hoboken, NJ, 2007.

[38] T. Ross, Fuzzy Logic with Engineering Applications, 3rd ed., John Wiley and Sons, West Sussex, UK, 2010.

[39] M. Hanss, Applied Fuzzy Arithmetic: An Introduction with Engineering Applications, Springer, Berlin, 2005.

[40] K.P. Lin, M.J. Wu, K.C. Hung, and Y. Kuo, "Developing the Weakest T-Norm Fuzzy GERT for Evaluating Uncertain Process Reliability in Semiconductor Manufacturing", Applied Soft Computing, vol. 11, no. 8, pp. 5165-5180, 2011. 